Europhysics Letters

PREPRINT

\title{
Poling effect on distribution of quenched random fields in a uniaxial relaxor ferroelectric
}

\author{
Manuel I. Marqués ${ }^{1}$ and Carmen Aragó ${ }^{1}$ \\ 1 Departamento de Física de Materiales C-IV, Universidad Autónoma de Madrid, 28049 \\ Madrid, Spain
}

PACS. 77.80.Bh - Phase transitions and Curie Point.

PACS. 77.80.-e - Ferroelectricity and antiferroelectricity.

PACS. 64.60.Fr - Equilibrium properties near critical points, critical exponents.

\begin{abstract}
The frequency dependence of the dielectric permitivity's maximum has been studied for poled and unpoled doped relaxor strontium barium niobate $\mathrm{Sr}_{0.61} \mathrm{Ba}_{0.39} \mathrm{Nb}_{2} \mathrm{O}_{6}$ : $\mathrm{Cr}^{3+}$ (SBN-61:Cr). In both cases the maximum found is broad and the frequency dispersion is strong. The present view of random fields compensation in the unpoled sample is not suitable for explaining this experimental result. We propose a new mechanism where the dispersion of quenched random electric fields, affecting the nanodomains, is minimized after poling. We test our proposal by numerical simulations on a random field Ising model. Results obtained are in agreement with the polarization's measurements presented by Granzow et al. [Phys. Rev. Lett 92, 065701 (2004)].
\end{abstract}

Relaxor ferroelectrics were discovered almost fifty years ago [1] and they have recently found a multitude of technical applications [2]. In particular, from the point of view of optical devices, uniaxial strontium barium niobate (SBN) has been proved to be extremely useful $[3,4]$. Relaxor ferroelectrics present a transition with temperature from polar to nonpolar phase which is characterized by a broad, frequency dependent maximum of the dielectric permitivity [5]. Another important feature of the relaxor ferroelectrics is the existence of locally polar nanoregions well above the Curie temperature $T_{C}$ which persist until the socalled Burns temperature $T_{B}[6]$, which is close to $350^{\circ} \mathrm{C}$ in $\mathrm{SBN}[7]$.

In SBN, these properties have been studied taking into account the inherent disorder of the relaxor $[8,9]$ by means of a random field Ising model (RFIM) [10]. The disorder produces quenched local random fields which interact with local dipoles. The local random fields stabilize the dipoles in a small nanoregion, leading locally to a nonzero value of the spontaneous polarization even above the Curie temperature. The use of a RFIM to model uniaxial ferroelectrics has been supported by recent findings in SBN which point toward the existence of internal fields [11-13].

Based on the RFIM, it is possible to make predictions about critical exponents in SBN. Theoretical calculations for the RFIM predict small values for the spontaneous polarization critical exponent $\beta=0.06 \pm 0.07$ [14]. Due to this small value, the decrease of polarization

(C) EDP Sciences 
when increasing temperature close to the Curie point must be very sharp compared with the behavior of other systems such as Ising models, where $\beta \simeq 0.325$.

Actually, there has been some controversy about the value of $\beta$ for SBN crystals. NMR measurements yielded $\beta=0.14$ [15] very close to the one predicted by the RFIM. However, linear birefringence measurements in SBN resulted in $\beta \sim 0.35$ [16], which is actually closer to the value of the Ising model. This discrepancy has been recently solved by pointing out that NMR samples where fully poled, while linear birefringence samples where unpoled [17]. Granzow et al. have shown experimentally how the critical exponent $\beta$ varies from $\beta=0.3$ in the case of a non-poled sample to $\beta=0.126$ when the sample is fully poled. This is equivalent to say that polarization decreases faster when increasing temperature in poled SBN than in non-poled SBN.

What is the reason for this behavior? A possible answer came from Ref. [17]: Basically, quenched random fields are compensated by those emerging from charged fractal nanodomain walls in the unpoled sample. Since there is no random electric field, the behavior of the system resembles the one of an Ising model. However, when SBN is poled, the compensation of the electric fields is not so effective due to the larger size of ferroelectric domains. Since the electric fields do not dissapear, the behavior for the poled sample must be the one corresponding to a RFIM.

Assuming that quenched random fields are responsible for the relaxor behavior in SBN, a sharper dielectric peak and a decrease in the frequency dispersion should be expected for the unpoled sample. To check this hypothesis we have performed dielectric permitivity measurements in SBN-61:Cr for poled and unpoled samples.

The SBN-61:Cr unpoled samples were small platelets of thickness $0.8 \mathrm{~mm}<d<1.8 \mathrm{~mm}$ and areas between $12 \mathrm{~mm}^{2}$ and $48 \mathrm{~mm}^{2}$. They were doped with a small amount $(0.01 w t \%)$ of $\mathrm{Cr}^{3+}$, but it seems that the impurity type is more relevant from the optical than the ferroelectric point of view. The two faces perpendicular to the ferroelectric axis were covered with electro-conductive silver paint. The samples have been grown by Czocharalski technique at the General Physics Institute of the RAS, Laser Materials and Technologies Research Center, Moscow, Russia. The measurements of the dielectric constant were performed under $1 \mathrm{~V}$ of applied voltage, with a LCR meter HP 4284A whose frequency ranges from $20 \mathrm{~Hz}$ to $1 \mathrm{MHz}$. The temperature control was made through a Unipan thermal controller type 680, provided with a sensor whose accuracy is $0.1 K$ and the sample actual temperature was obtained with a T thermocouple and a DMM Keithley 196. The poled sample was measured using the following procedure: Once the system reached a temperature higher than the critical temperature, we pre-polarized the sample applying a constant $2.8 \mathrm{kV} / \mathrm{cm}$ DC field while freezing the sample until RT and then the dielectric constant measurements were performed for the same frequencies that in the unpoled case.

The experimental measurements presented in Fig.1 show the typical relaxor frequency and temperature dependence of dielectric constant, with a rounded peak and a non-well defined transition temperature. So actually, both samples behave in a very similar way from the relaxor behavior point of view. The value of the peak of dielectric constant we find for SBN-61:Cr is much smaller than the one found in Ref. [18] for SBN-61:Ce. This small value may be due to the quality of the sample or to the fact that $C r$ doping increases the SBN relaxor behavior. In any case, both materials present relaxor properties for poled and unpoled samples.

It is not easy to explain the unpoled results when considering random fields compensation in the unpoled sample. The broad maximum and the frequency dispersion found for the nonpoled sample imply that quenched random fields are not compensated. On the other hand, the broad maximum also appears for the poled sample, indicating that random fields are present 


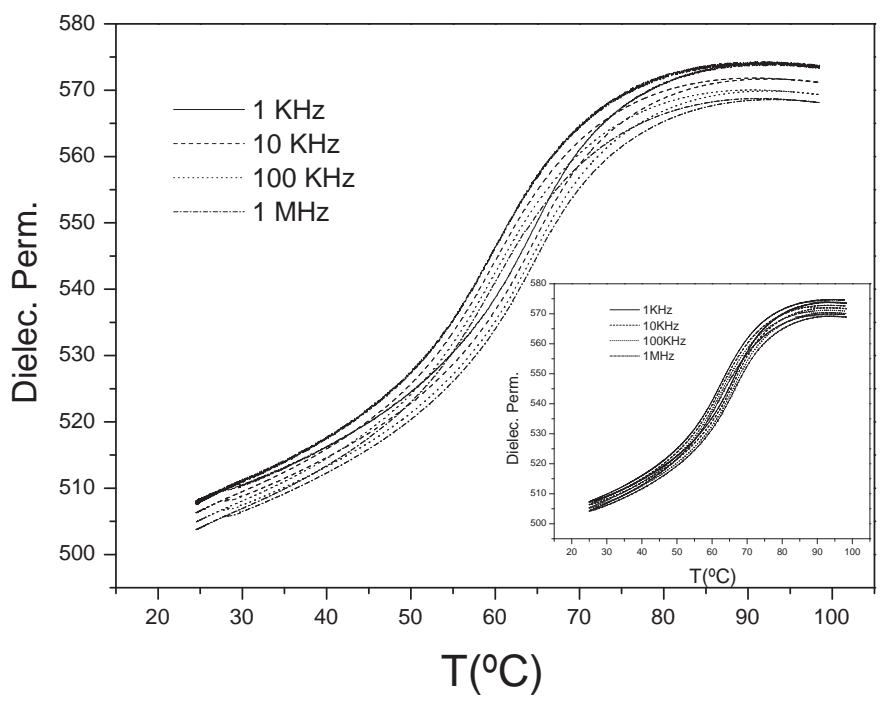

Fig. 1 - Dielectric permitivity vs. temperature for non-poled sample of SBN-61:Cr. Inset: poled sample

too. However, there must be some differences between the fields distributions when comparing both samples, in order to explain the changes observed in the polarization behavior [17]. How to explain the dielectric permitivity experimental results and how to make them compatible with the ones observed for the behavior of spontaneous polarization versus temperature in Ref. [17]?

In the following we will propose a model to answer this question. Local fields are due to positive charges either in deficit at unoccupied $\mathrm{Ba}^{2+}$ and $\mathrm{Sr}^{2+}$ sites in the open tungsten bronze structure [19], or in excess at $\mathrm{Cr}^{3+}$ ions replacing $\mathrm{Nb}^{5+}[20]$.

The fields are quenched due to the low mobility of the charge carriers below the Curie temperature [21] and they are almost uncorrelated due to the short-range character coming from a large dielectric dc susceptibility [9]. An electric field applied to polarize the SBN sample at temperatures below the transition point should interact with the charge carriers, introducing some degree of correlation between them [22], and decreasing the value of the dispersion of local fields. Once the electric field is turned off, the new "ordered" internal electric fields become quenched at room temperature. Of course, a change on the dispersion $\sigma$ of the Gaussian distribution of fields in a RFIM does not change the critical behavior of the system (only the critical temperature) [14] so, a change on $\beta$ is not expected. However, we should take into account that nanodomains found in relaxors at high temperatures are not formed by just a single dipole. It is necessary to consider that a quenched randon field affects not just to a single dipole but to a local group of dipoles forming a nanodomain. A change on the dispersion of local fields affecting complete nanodomains may change the behavior with temperature of the total polarization of the system.

We propose the following hypothesis: (i) A poled sample presents a distribution of nanodomain electric fields with an small dispersion. Due to this small dispersion, all the nan- 


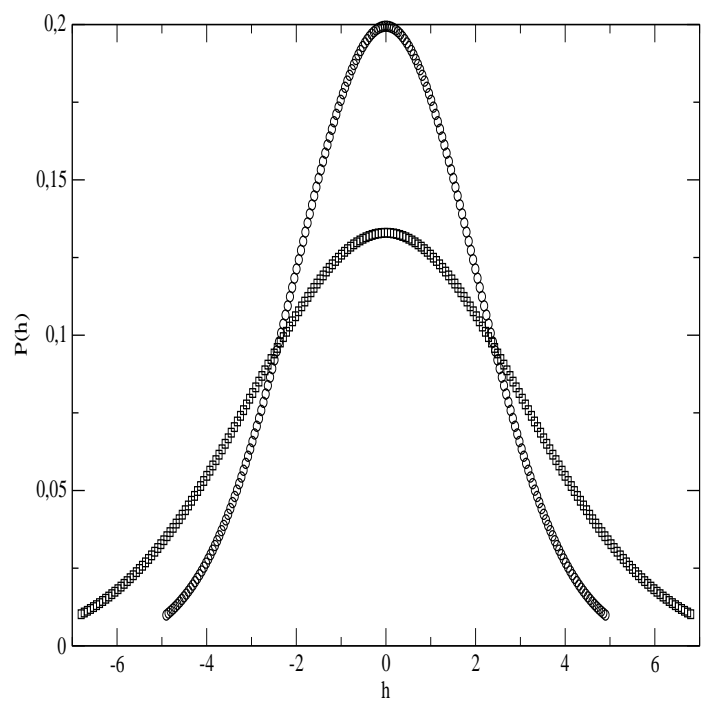

Fig. 2 - Distribution $P\left(h_{\alpha}\right)$ of fields for $\sigma=2$, corresponding to the poled sample (circles) and for $\sigma=3$ corresponding to the un-poled sample (squares).

odomains behave similarly when the critical temperature is approached and they decrease their polarization almost simultaneously. This commom behavior implies a sharp transition for the total polarization versus temperature ( $\beta$ decreases). (ii) A non-poled sample presents a distribution of nanodomain electric fields with a huge dispersion. When the critical temperature is approached the behavior of the nanodomains is very different from one to the other and the local polarizations do not decrease simultaneously. This behavior results on a smooth behavior of the total polarization versus temperature ( $\beta$ increases). However, in both cases, nanodomain electric fields are not compensated explaining the broad peak and frequency dependence found in experiments for both, poled and unpoled samples.

To check this hypothesis we present Monte Carlo simulations of several random field Ising models, with a Gaussian distribution of fields centered in zero and with a width of the distribution given by $\sigma$. We will consider different values of $\sigma$, corresponding to poled and unpoled samples of SBN. To study the effect of a random field affecting not just to a single dipole but to a group of dipoles, we divide the system in several nanoregions $\alpha$, assigning a single electric field to all dipoles belonging to the same nanoregion. To make calculation easier, we consider a cubic system formed by $N^{3} \times L^{3}$ dipoles with periodic boundary conditions formed by small cubic-shaped nanoregions with $N^{3}$ dipoles. The Hamiltonian reads as follows:

$$
H=-J \sum_{<i j>} s_{i} s_{j}-J_{h} \sum_{\alpha} \sum_{i \in \alpha} h_{\alpha} s_{i}
$$

and the distribution of quenched fields is given by,

$$
P\left(h_{\alpha}\right)=\frac{1}{\sqrt{2 \pi} \sigma} \exp \left[-\frac{h_{\alpha}^{2}}{2 \sigma^{2}}\right]
$$

The distributions of fields considered in this work for the poled and the non-poled samples are shown in Fig.2 


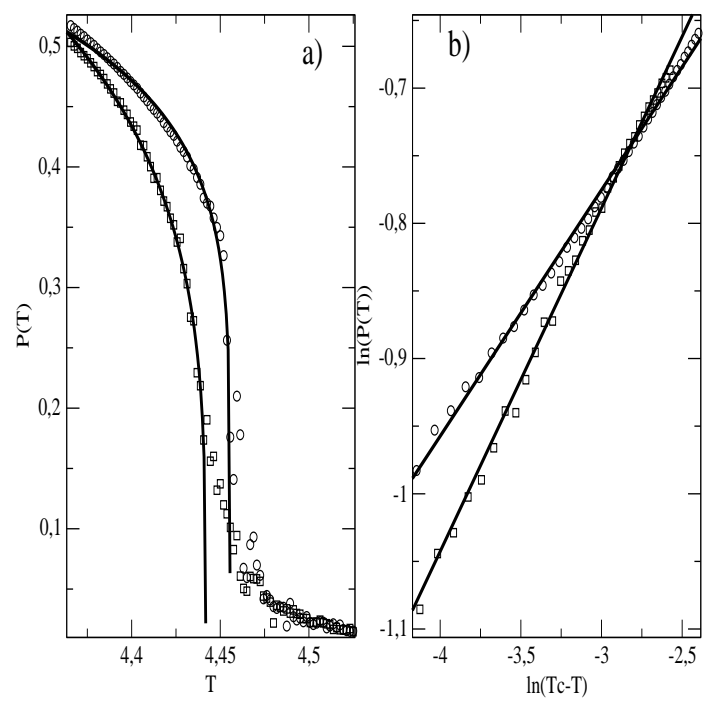

Fig. 3 - (a) Polarization $P(T)$ vs. temperature $T$ for $\sigma=2$, corresponding to the poled sample (circles) and for $\sigma=3$ corresponding to the un-poled sample (squares). Continuous lines represent a fitting to a power law. (b) $\ln (P(T))$ vs. $\ln \left(T_{c}-T\right)$ for $\sigma=2$, corresponding to the poled sample (circles) and for $\sigma=3$ corresponding to the un-poled sample (squares). Continuous lines represent a linear fitting.

For our simulations we set the values $N=4, L=25, J=1$ and $J_{h}=0.02$. In Fig.3a we present results for the total normalized polarization $P=\left[1 /\left(N^{3} \times L^{3}\right)\right] \sum_{i} s_{i}$ versus temperature for $\sigma=2$ (representing the poled sample) and $\sigma=3$ (representing the unpoled sample). We have fitted the data to a power law $P(T)=P_{0}\left(1-T / T_{c}\right)^{\beta}$ for $T<T_{c}$. The values obtained are $T_{c}=4.45$ and $\beta=0.17$ for $\sigma=2$ and $T_{c}=4.44$ and $\beta=0.26$ for $\sigma=3$. These results for $\beta$ compare with the ones obtained experimentally for SBN in ref. [17] for a poled sample (with initial spontaneous polarization $P\left(20^{\circ} \mathrm{C}\right) \sim 10\left(\mu \mathrm{C} / \mathrm{cm}^{2}\right)$ ) and a non-poled sample respectively. Fig.3b presents the power law fits in log scale.

There is a difference between the model we are proposing and the former RFIM studied in detail by Newman and Barkema [14]. In the former RFIM each single dipole was influenced by a different random field and a change on $\sigma$ affected to the randomness of the system but did not affect to the critical behavior. In contrast, in the present model, the same random field affects to a group of $N \times N \times N$ dipoles forming a nanoregion. These nanoregions may act almost independently from each other (as a collection of non-random Ising models) if the differences on the values of the fields are large (i.e. if $\sigma$ is large), changing the critical behavior from RFIM to Ising model. The value we find for $\beta$ in the case of a poled sample is very similar to the one observed experimentally yet not exactly equal to the value $(\beta<0.06 \pm 0.07)$ reported for the RFIM in Ref. [14], but it would become very similar in the special case of considering very small nanoregions $(N=1)$.

It is also possible to understand this difference on the critical behavior of the polarization by considering the polarization of each single nanodomain versus the temperature. Results are presented in Fig.4 for 50 different nanodomains. Note how, in both cases, the polarization for each nanodomain remains different from zero at $T>T_{c}$, as usual for a relaxor. However, the dispersion on the polarization curves for the poled $\sigma=2$ case (Fig.4a) is smaller than the one we find for the unpoled $\sigma=3$ case (Fig.4b). The critical temperature for each nanodomain 
EUROPHYSICS LETTERS

may be determined by considering the maximum of the derivative of the polarization in each nanoregion versus the temperature. A seasonal derivative with a period of ten points is shown in Fig.4a and Fig.4b. For a poled sample, all nanodomains present their critical temperatures in a narrow window around the critical temperature of the system. Since all nanodomains experiment the transition at almost the same temperature, the behavior of the spontaneous polarization is very sharp and the value of $\beta$ very small. On the other hand, when the sample is non-poled, the critical temperatures of the nanodomains are distributed on a wider temperature range. This makes the total polarization not to decrease abruptly, obtaining a larger value of $\beta$ than the one corresponding to the poled sample. Relaxor behavior is expected in both cases, since electric fields are never compensated.

In Ref. [17] samples are polarized at room temperature, but it is also posible to polarize at high temperature and to perform field cooling (FC) [12]. In this special case, the relaxor exhibits an stable polarization at the cooling process with zero field applied. This experimental results are explained by Granzow et al. as follows: At high temperatures electric charge carriers are very mobile and they are able to travel through the crystal and compensate the local random electric fields not in accordance with the externally applied electric field. This process imprints an stable non-zero internal field capable of polarizing the sample at low temperatures. We have measured the critical exponents from the experimental results presented for the SBN relaxor at Ref. [12] and we have found a value of $\beta=0.15$ for the heating process and a value $\beta=0.25$ for the cooling process. Our proposal is supported also by these results obtained for FC samples. From the point of view of our model, the field compensation coming from the travelling charges at the FC process implies a non-centered gaussian distribution of fields (a total value of the internal electric field different from zero). Also, the relaxor will show a short range ordering as the one already described for the poling process at room tepratures [22], turning intto a decrease on the value of $\sigma$ and the value of $\beta(\beta=0.15)$. Once the system is heated to very hight temperatures, the internal electric field still remains [12], but the short range ordering dissapears ( $\sigma$ turns broad again). Then ,at the cooling process with no external field applied, the system will show the spontaneous polarization comming from a non-centered distribution of internal fields, with a broad value of $\sigma$, turning into a large value of $\beta(\beta=0.25)$. Note how, in this case, it is imposible to explain this large $\beta$ value by a total compensation of the internal fileds (pure Ising model), since non-zero internal fields are needed to explain the existence of spontaneous polarization when cooling.

In summary, the dielectric dispersion in relaxor SBN is similar for the non-poled and for the poled sample. For this reason, an explanation for the change on the critical behavior of polarization based on fields compensation in the unpoled sample is not appropriate. A mechanism capable of explaining both behaviors has been proposed. In this new mechanism a poled sample presents an smaller dispersion of quenched electric fields than a non-poled sample. Monte Carlo simulations show how this model leads to a different behavior of the local polarization in the nanodomains of the relaxor system. This difference on behavior produces a change on the criticality of the total polarization similar to the one found experimentally.

We thank M.Ramirez and J.García Solé for supplying the samples. Helpful discussions with J.A.Gonzalo and W.Kleemann are greatfully acknowledged. This work was supported by the DGICyT through grant BFM2000-0032. 

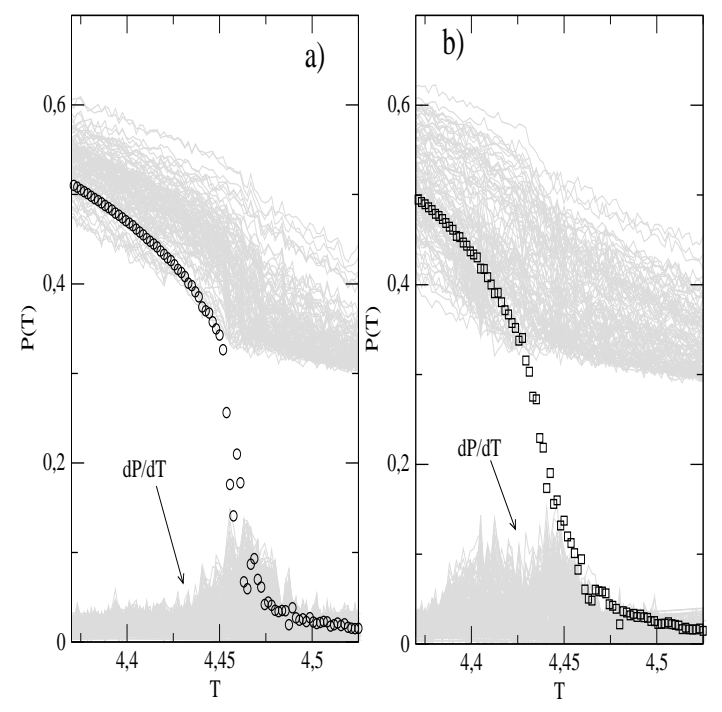

Fig. 4 - Polarization $P(T)$ vs. $T$ for (a) $\sigma=2$, corresponding to the poled sample (circles) and (b) $\sigma=3$, corresponding to the non-poled sample (squares). Grey lines represent polarization of each nanodomain $\left(P_{\alpha}\right)$ and $d P_{\alpha} / d T$ vs. temperature for 50 different nanodomains.

\section{REFERENCES}

[1] G. A. Smolenskii and A. I. Agranovskaya, Fiz. Tverd. Tela 1, 1562 (1958); G. A. Smolenskii et al., Sov. Phys. Solid State 2, 2584 (1961).

[2] K. Uchino, Piezoelectric Actuators and Ultrasonic Motors (Kluwer Acadmic, Boston, 1996).

[3] M. J. Miller et al., Opt. Lett. 12, 340 (1987).

[4] X. Yue at al., Opt. Lett. 17, 142 (1992).

[5] See f.i. G. A. Samara, J. Phys: Condens. Matter 15, R367 (2003).

[6] G. Burns and F. H. Dacol, Phys. Rev. B 28, 2527 (1983).

[7] P. Lehnen, W. Kleemann, Th. Woike and R. Pankrath, Eur. Phys. J. B 14, 633 (2000).

[8] W. Kleemann, Int. J. Mod. Phys. B 7, 2469 (1993).

[9] W. Kleemann et al., Europhys. Lett. 57, 14 (2002).

[10] Y. Imry and S. Ma, Phys. Rev. Lett. 35, 1399 (1975).

[11] T. Granzow et al., Europhys. Lett. 57, 597 (2002).

[12] T. Granzow et al., Appl. Phys. Lett. 80, 470 (2002).

[13] T. Granzow et al., Phys. Rev. Lett. 89, 127601 (2002).

[14] M. E. J. Newman and G. T. Barkema, Phys. Rev. E 53, 393 (1996).

[15] R. Blinc et al., Phys. Rev. B 64, 134109 (2001).

[16] P. Lehnen et al., Euro. Phys. J. B 14, 633 (2000).

[17] T. Granzow et al., Phys. Rev. Lett 92, 065701 (2004).

[18] J. Dec, W. Kleemann, Th. Woike and R. Pankrath, Eur. Phys. J. B 14, 627 (2000).

[19] A. M. Glass, J. Appl. Phys. Rev. 40, 4699 (1969).

[20] T. Woike et al., Appl. Phys. B 72, 661 (2001).

[21] K. Buse, U. van Stevendaal, R. Pankrath, and E. Krätzing, J. Opt. Soc. Am. B 13, 1461 (1996).

[22] J. Dec et al. Phys. Rev. B 68, 092105 (2003). 